Structure-Directing Effects of Coordinating Solvents, Ammonium and Phosphonium Counterions in Uranyl Ion Complexes with

\title{
1,2-, 1,3- and 1,4-Phenylenediacetates
}

Pierre Thuéry, ${ }^{*, \dagger}$ Youssef Atoini ${ }^{\sharp}$ and Jack Harrowfield*,\$

†NIMBE, CEA, CNRS, Université Paris-Saclay, CEA Saclay, 91191 Gif-sur-Yvette, France †ISIS, Université de Strasbourg, 8 allée Gaspard Monge, 67083 Strasbourg, France

Supplementary Information

(a) 1,2-pda ${ }^{2-}$ complexes
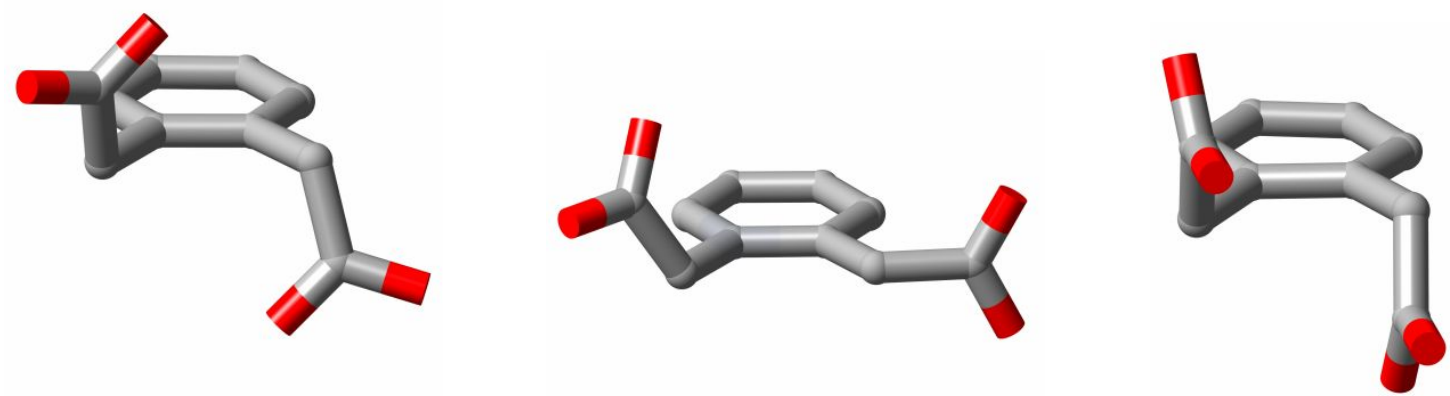

$\left[\mathrm{UO}_{2}(1,2-\right.$ pda $\left.)\right] \cdot \mathrm{CH}_{3} \mathrm{CN}(\mathbf{1})$

$\left[\mathrm{UO}_{2}(1,2-\right.$ pda)(DMPU) $](3) \quad\left[\mathrm{UO}_{2}(1,2-\right.$ pda)(bipy) $] \cdot \mathrm{CH}_{3} \mathrm{CN}$

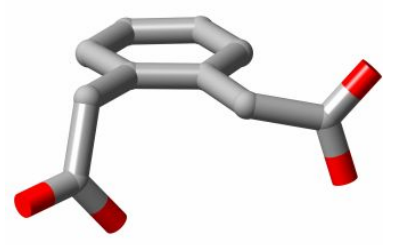

$\left[\mathrm{UO}_{2}(1,2-\mathrm{pda})(\mathrm{phen})\right]$

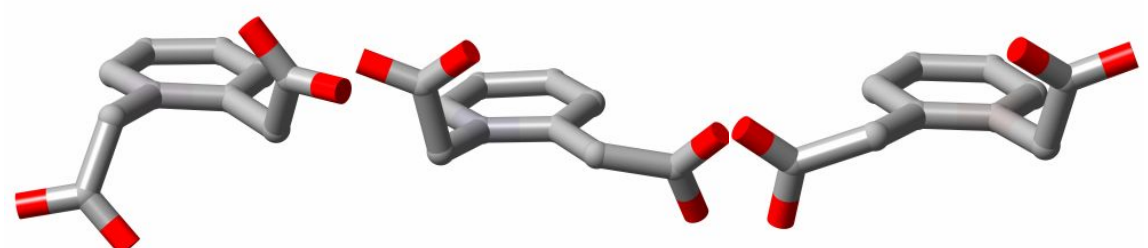

ligand 1 ligand 3

$$
\left[\mathrm{H}_{2}-2.2 .2\right]\left[\left(\mathrm{UO}_{2}\right)_{2}(1,2-\mathrm{pda})_{3}\right] \cdot \mathrm{CH}_{3} \mathrm{CN}
$$




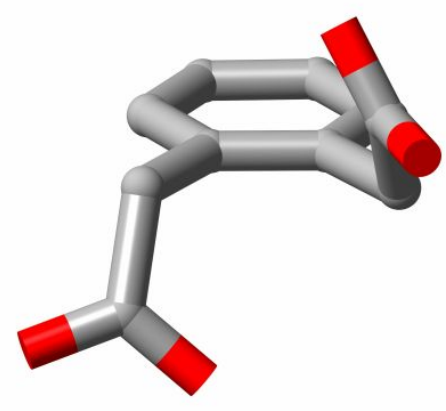

ligand 1

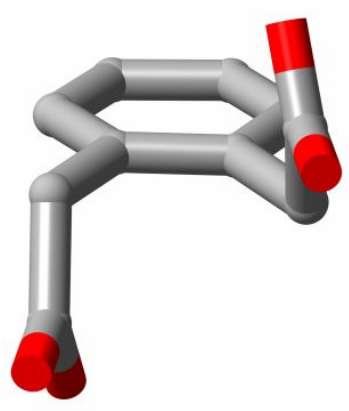

ligand 2

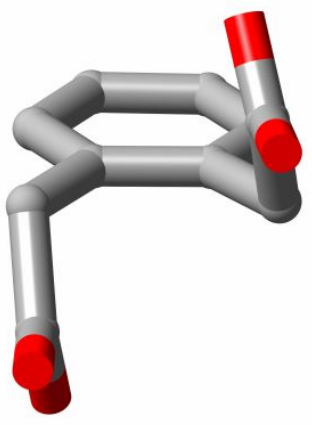

ligand 3

$\left[\mathrm{H}_{2} \mathrm{NMe}_{2}\right]_{2}\left[\left(\mathrm{UO}_{2}\right)_{2}(1,2-\mathrm{pda})_{3}\right] \cdot \mathrm{H}_{2} \mathrm{O}(7)$

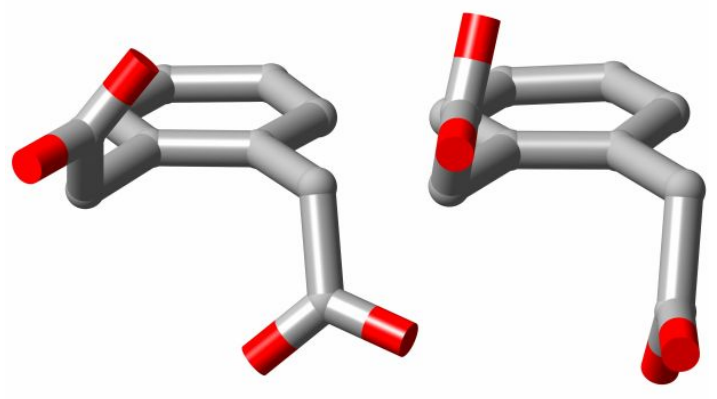

$\left[\mathrm{H}_{2} \mathrm{NMe}_{2}\right]_{2}\left[\left(\mathrm{UO}_{2}\right)_{2}(1,2-\mathrm{pda})_{3}\right] \cdot 3 \mathrm{H}_{2} \mathrm{O}(\mathbf{8})$
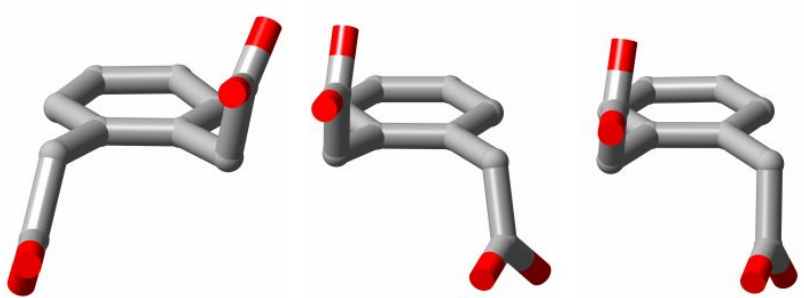

ligand 3

ligand 1

ligand 2

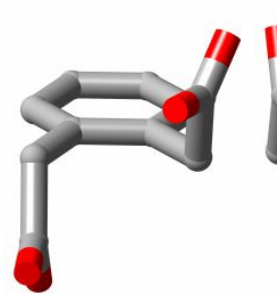

ligand 4 ligand 5

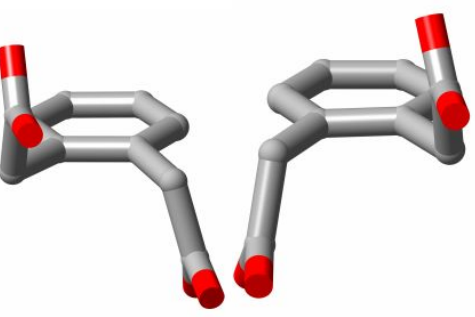

ligand 6

$\left[\mathrm{C}\left(\mathrm{NH}_{2}\right)_{3}\right]_{2}\left[\left(\mathrm{UO}_{2}\right)_{2}(1,2-\mathrm{pda})_{3}\right] \cdot 0.5 \mathrm{H}_{2} \mathrm{O} \cdot \mathrm{CH}_{3} \mathrm{CN}(\mathbf{1 2})$

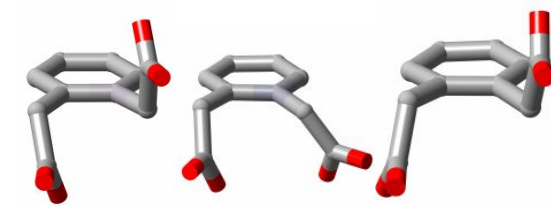

ligand 1 2 3

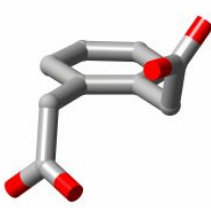

4

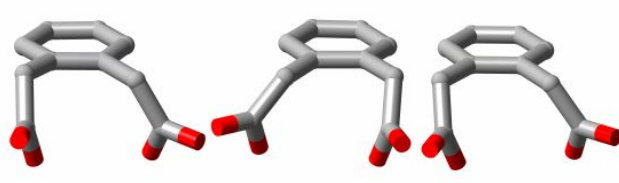

5
6

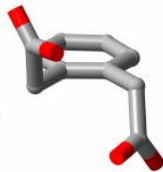

8

$\left[\mathrm{NH}_{4}\right]_{6}\left[\mathrm{Ni}\left(\mathrm{H}_{2} \mathrm{O}\right)_{6}\right]_{2}\left[\left(\mathrm{UO}_{2}\right)_{4}(1,2-\mathrm{pda})_{6}\right]_{2}\left[\left(\mathrm{UO}_{2}\right)_{4}(1,2-\mathrm{pda})_{5}\left(\mathrm{H}_{2} \mathrm{O}\right)_{4}\right]$ 


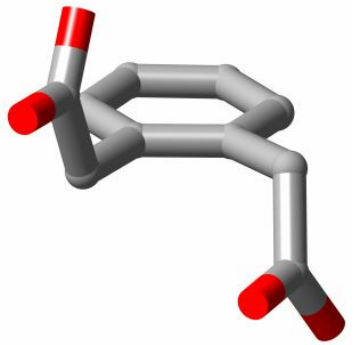

$\left[\mathrm{Cu}(\text { bipy })_{2}\left(\mathrm{NO}_{3}\right)\right]\left[\mathrm{UO}_{2}(1,2-\mathrm{pda})\left(\mathrm{NO}_{3}\right)\right]$
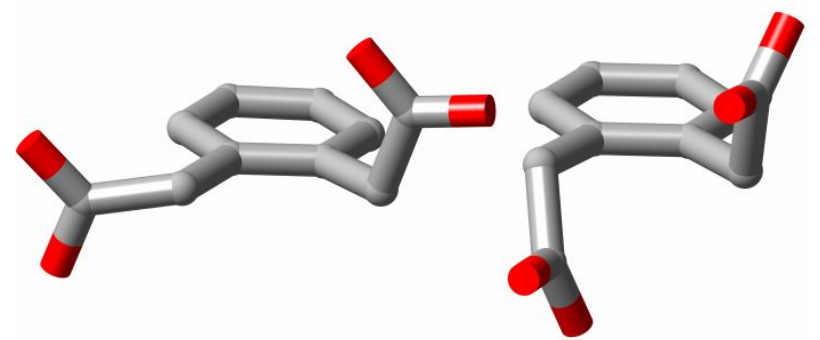

ligand UU

ligand UZn

$\left[\mathrm{UO}_{2}(1,2-\text { pda })_{2} \mathrm{Zn}(\text { phen })_{2}\right] \cdot 2 \mathrm{H}_{2} \mathrm{O}$

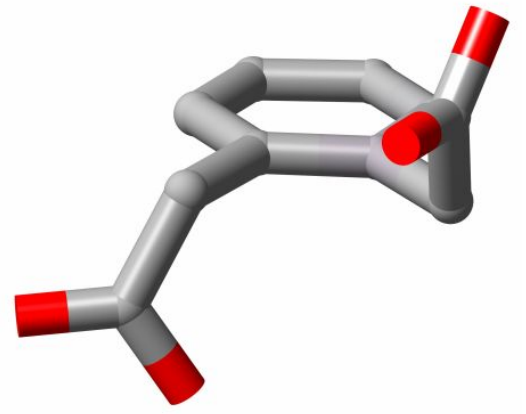

ligand 1

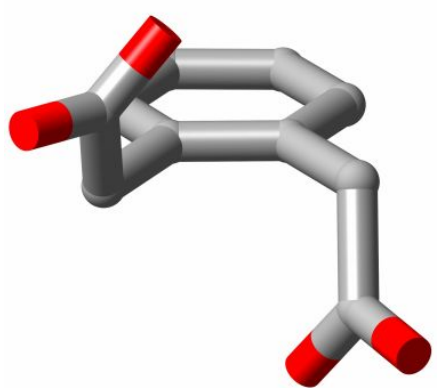

ligand 2

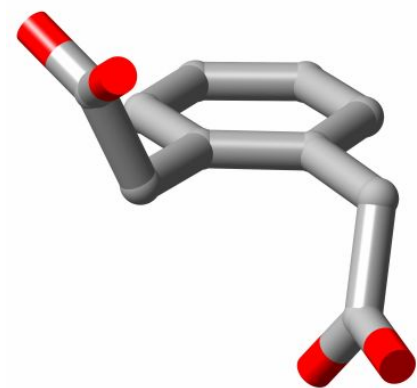

ligand 3

$\left[\mathrm{Zn}(\text { phen })_{3}\right]\left[\left(\mathrm{UO}_{2}\right)_{2}(1,2-\mathrm{PDA})_{3}\right] \cdot 7 \mathrm{H}_{2} \mathrm{O}$

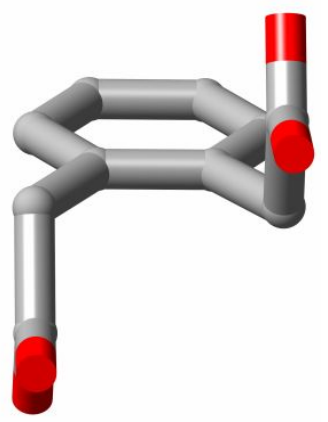

$\left[\mathrm{Ag}(\text { bipy })_{2}\right]\left[\mathrm{UO}_{2}(1,2-\right.$ pda $\left.)\left(\mathrm{NO}_{3}\right)\right]$

(b) 1,3-pda ${ }^{2-}$ complexes

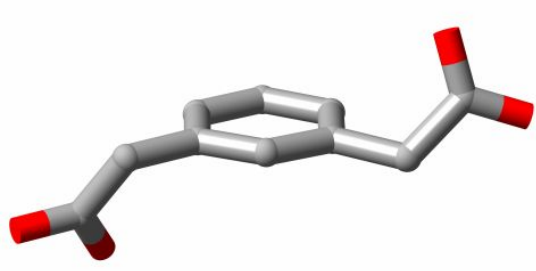

$\left[\mathrm{UO}_{2}(1,3-p d a)\right](2)$

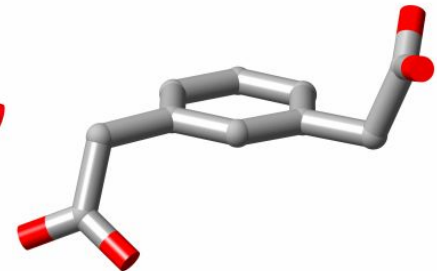

$\left[\mathrm{UO}_{2}(1,3-\mathrm{pda})(\mathrm{NMP})\right](4)$

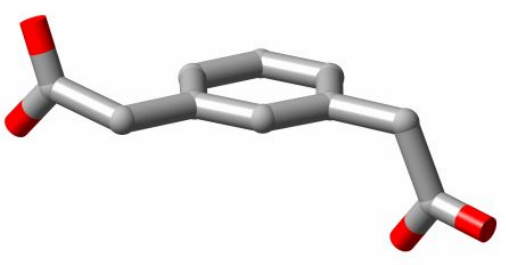

[UO 2 (1,3-pda)(bipy)] 

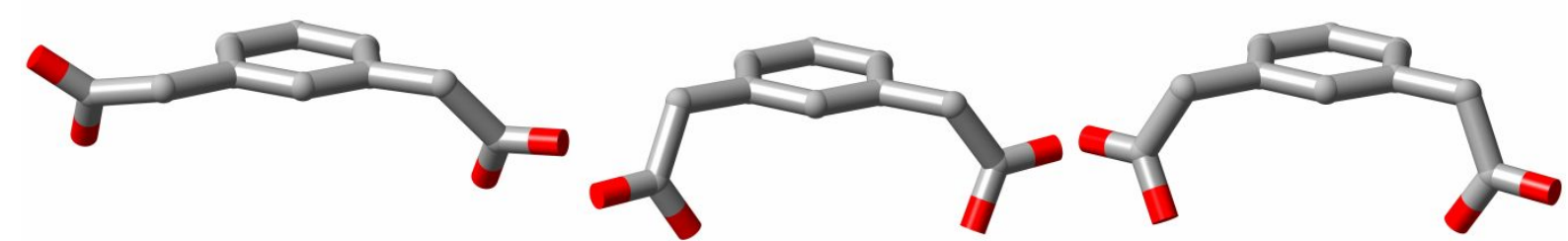

ligand 1

ligand 2

$\left[\mathrm{UO}_{2}(1,3-\mathrm{PDA})(\mathrm{phen})\right]$

$\left[\mathrm{H}_{2} \mathrm{NMe}_{2}\right]_{2}\left[\left(\mathrm{UO}_{2}\right)_{2}(1,3-\text { pda })_{3}\right] \cdot 0.5 \mathrm{H}_{2} \mathrm{O}(9)$
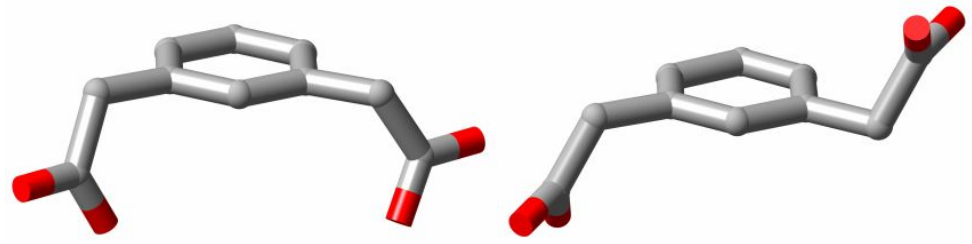

ligand 1

ligand 2

$\left[\mathrm{PPh}_{3} \mathrm{Me}\right]_{2}\left[\left(\mathrm{UO}_{2}\right)_{2}(1,3-\mathrm{pda})_{3}\right](\mathbf{1 3})$

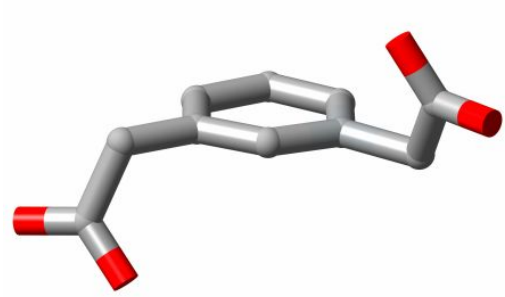

ligand 1

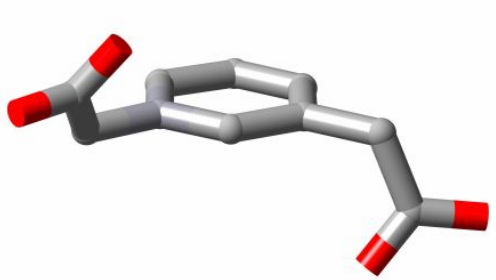

ligand 2

$[\mathrm{Ni}($ cyclam $)]\left[\left(\mathrm{UO}_{2}\right)_{2}(1,3-\mathrm{pda})_{3}\right]$

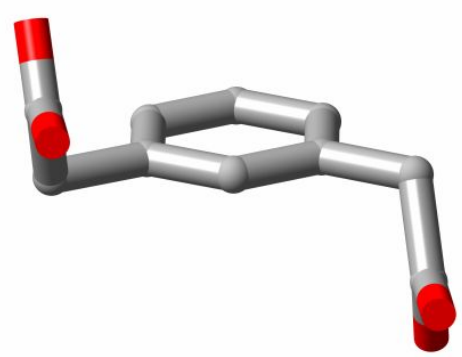

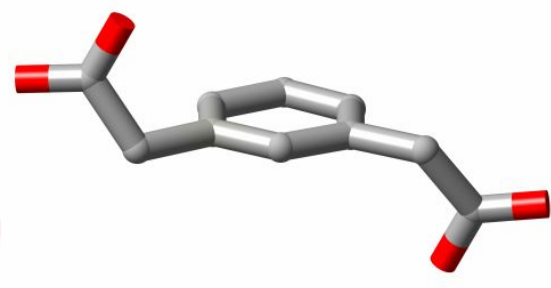

ligand 3

$\left[\mathrm{Cu}\left(R, S-\mathrm{Me}_{6}\right.\right.$ cyclam $\left.)\right]\left[\mathrm{UO}_{2}(1,3 \text {-pda })\left(\mathrm{NO}_{3}\right)\right]_{2}$ 


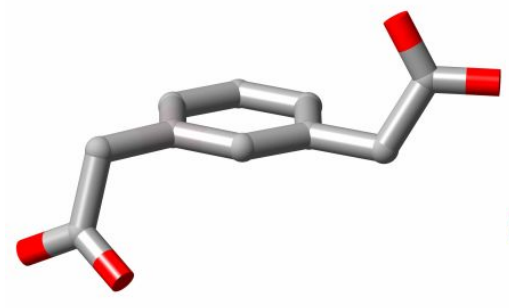

ligand 1

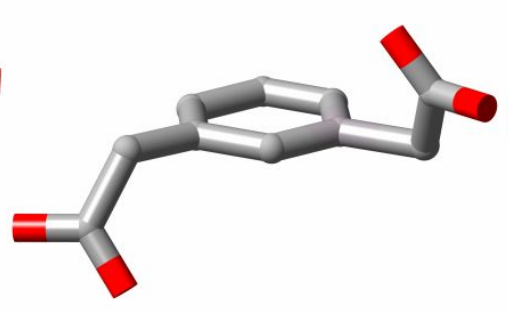

ligand 3

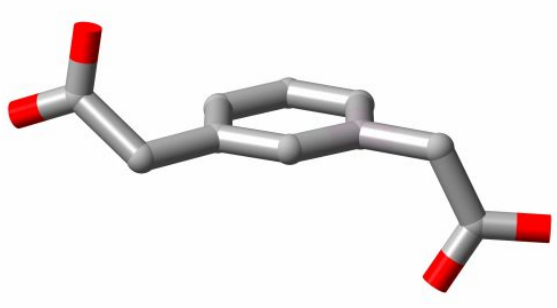

ligand 4

$$
\left[\mathrm{Zn}(\text { phen })_{3}\right]\left[\left(\mathrm{UO}_{2}\right)_{2}(1,3 \text {-pda })_{3}\right]
$$
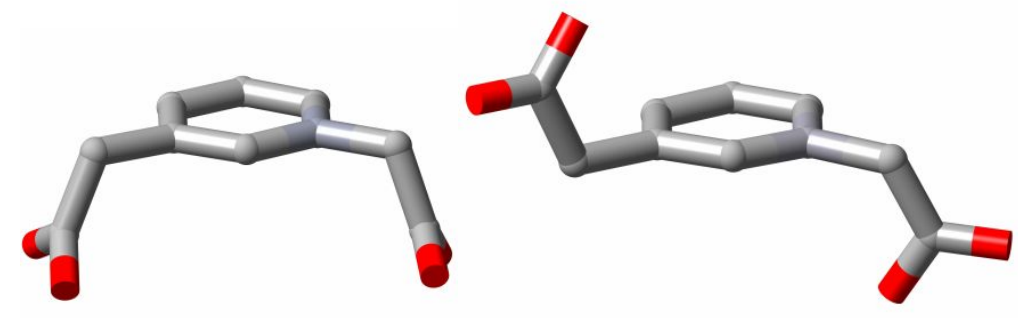

ligand 1

ligand 3

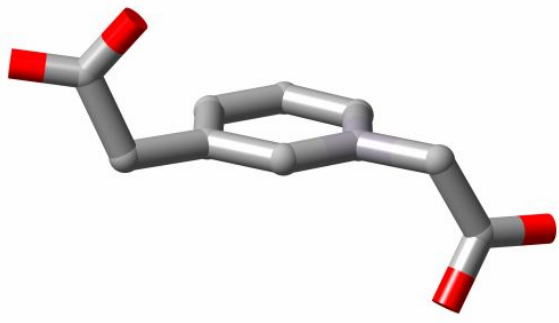

ligand 4

$\left[\mathrm{Zn}(\text { bipy })_{3}\right]\left[\left(\mathrm{UO}_{2}\right)_{2}(1,3-\mathrm{pda})_{3}\right]$

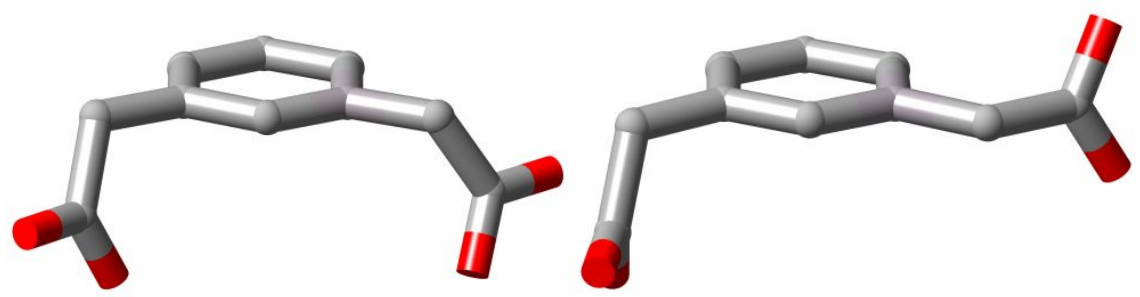

ligand 1

ligand 2

$\left[\mathrm{UO}_{2} \mathrm{~Pb}(1,3-\mathrm{pda})_{2}\right.$ (phen) $]$

(c) 1,4-pda ${ }^{2-}$ complexes

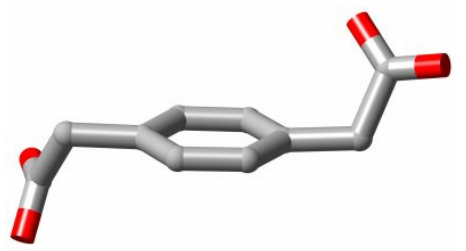

ligand 1

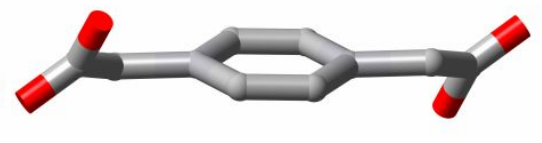

ligand 2

$\left[\mathrm{UO}_{2}(1,4-\mathrm{pda})(\mathrm{NMP})\right](\mathbf{5})$ [UO 2 (1,4-pda)(DMPU)] (6) 


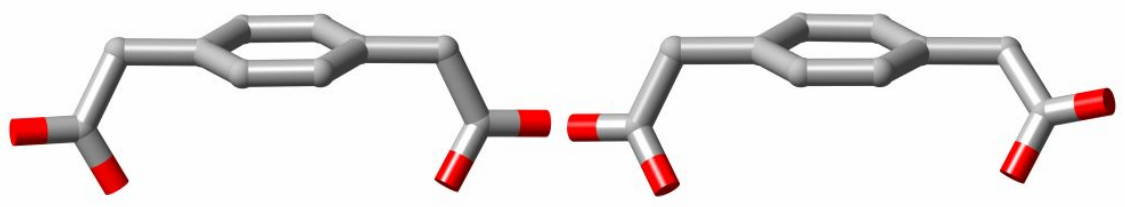

ligand 1

ligand 2

$\left[\mathrm{H}_{2} \mathrm{NMe}_{2}\right]_{2}\left[\left(\mathrm{UO}_{2}\right)_{2}(1,4-\mathrm{pda})_{3}\right](\mathbf{1 0})$

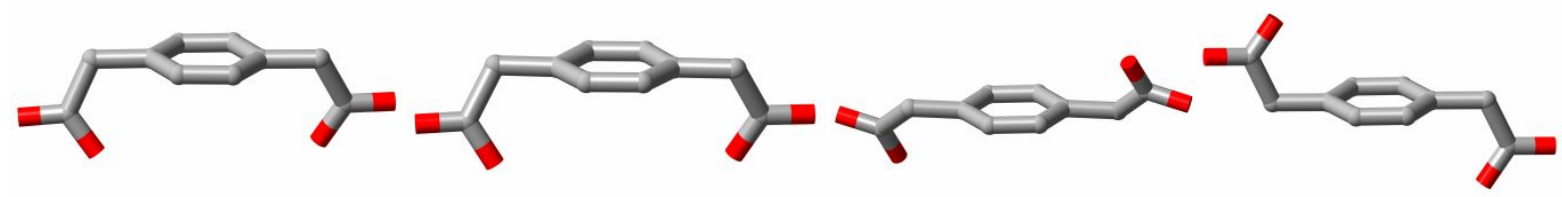

ligand 1

ligand 2

ligand 3

ligand 4

$\left[\mathrm{PPh}_{3} \mathrm{Me}\right]\left[\mathrm{H}_{2} \mathrm{NMe}_{2}\right]\left[\left(\mathrm{UO}_{2}\right)_{2}(1,4-\mathrm{pda})_{3}\right] \cdot \mathrm{H}_{2} \mathrm{O}(\mathbf{1 5})$

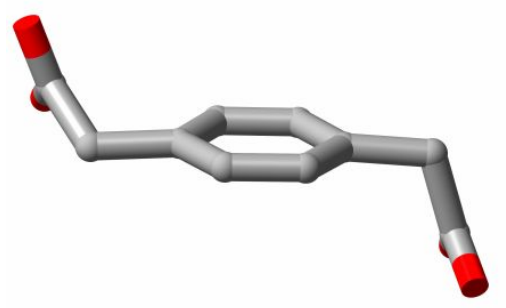

ligand 1

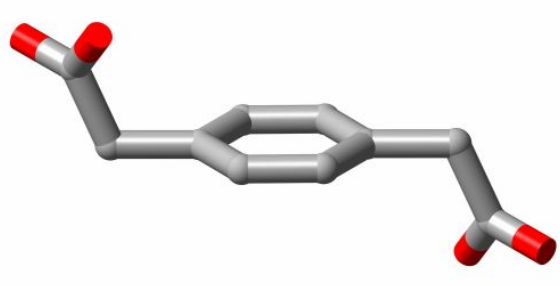

ligand 2

$\left[\mathrm{PPh}_{4}\right]_{2}\left[\left(\mathrm{UO}_{2}\right)_{2}(1,4-\mathrm{pda})_{3}\right](\mathbf{1 4})$

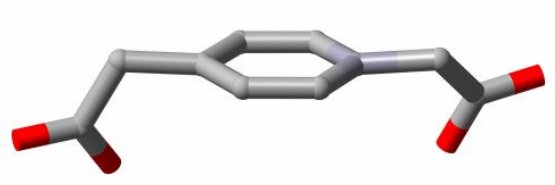

$\left[\mathrm{UO}_{2}(1,4-\text { pda })_{2} \mathrm{Mn}(\text { bipy })_{2}\right] \cdot \mathrm{H}_{2} \mathrm{O}$

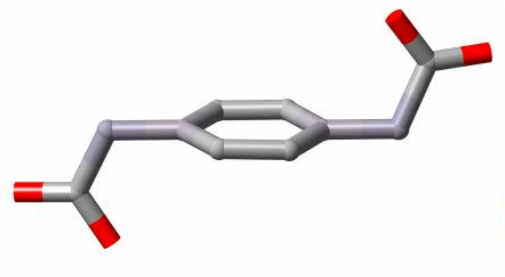

ligand 1

$\left[\mathrm{Co}(\text { bipy })_{3}\right]\left[\left(\mathrm{UO}_{2}\right)_{2}(1,4-\text { pda })_{3}\right]$

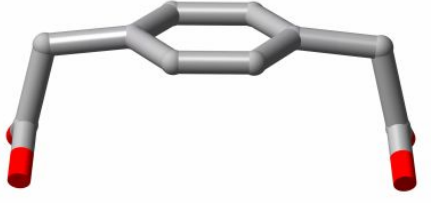

ligand 2 


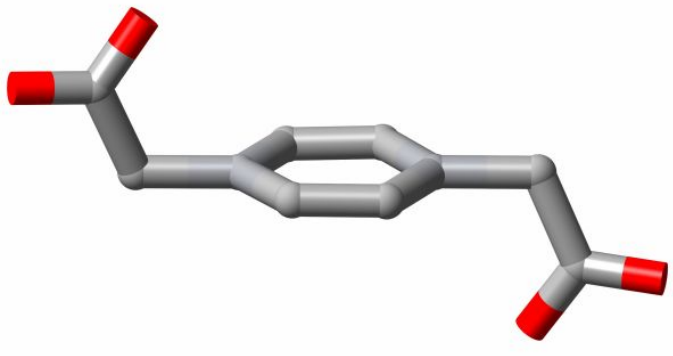

ligand 1

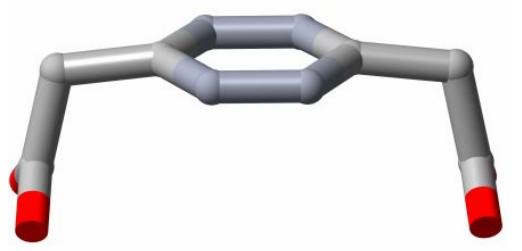

ligand 2

$\left[\mathrm{Ni}(\text { bipy })_{3}\right]\left[\left(\mathrm{UO}_{2}\right)_{2}(1,4-\mathrm{pda})_{3}\right]$

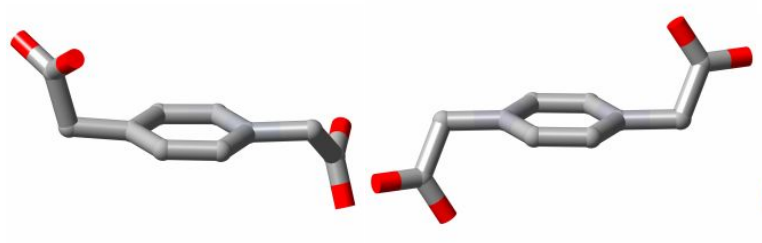

ligand 1

ligand 2

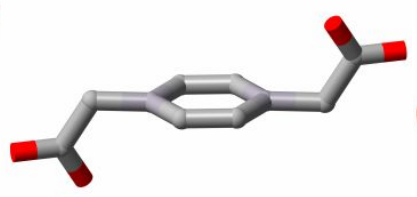

ligand 3

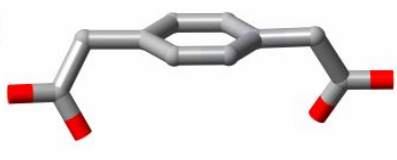

ligand 4

$\left[\mathrm{Ni}(\text { phen })_{3}\right]\left[\left(\mathrm{UO}_{2}\right)_{2}(1,4-\text { pda })_{3}\right] \cdot \mathrm{H}_{2} \mathrm{O}$

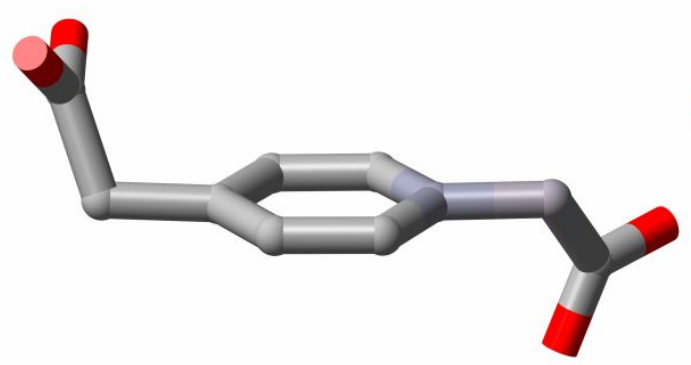

ligand 1

$\left[\mathrm{Cu}(\text { bipy })_{2}\right]\left[\mathrm{H}_{2} \mathrm{NMe}_{2}\right]\left[\left(\mathrm{UO}_{2}\right)_{2}(1,4-\mathrm{PDA})_{3}\right]$

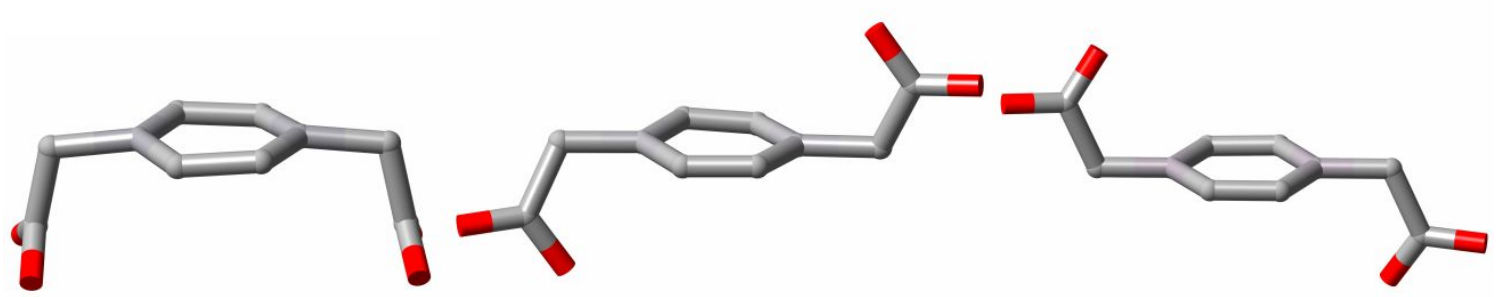

ligand 1

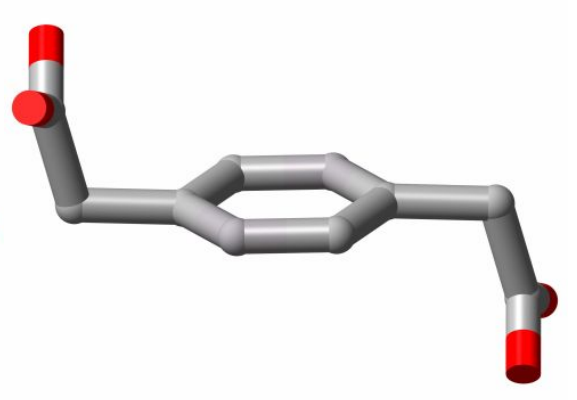

ligand 2

$$
\left[\mathrm{Cu}(\text { bipy })_{2}\right]\left[\mathrm{H}_{2} \mathrm{NMe}_{2}\right]\left[\left(\mathrm{UO}_{2}\right)_{2}(1,4-\mathrm{PDA})_{3}\right]
$$



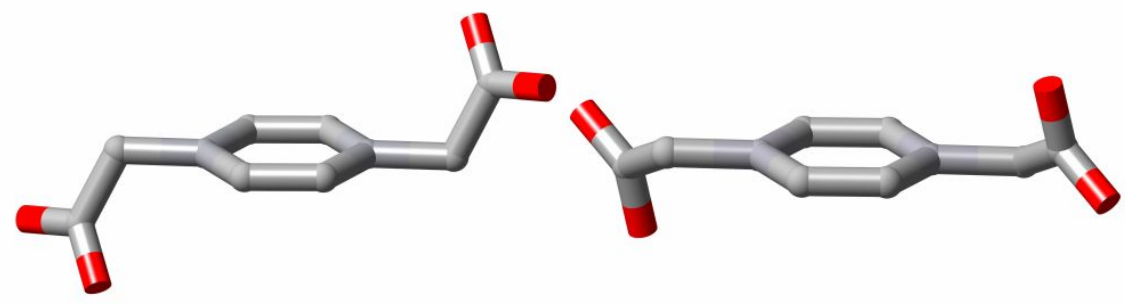

ligand 1

ligand 2

$\left[\mathrm{Ag}(\text { bipy })_{2}\right]\left[\mathrm{UO}_{2}(1,4-\right.$ pda $\left.)\left(\mathrm{NO}_{3}\right)\right]$

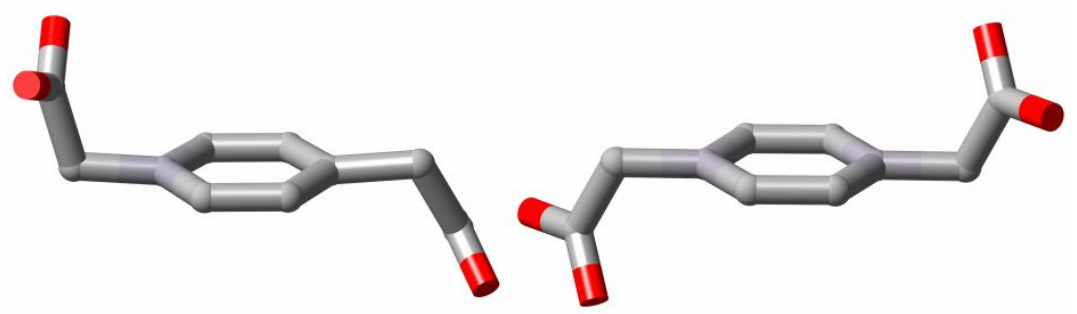

ligand 1

ligand 2

$\left[\left(\mathrm{UO}_{2}\right)_{2} \mathrm{~Pb}_{2}(1,4-\mathrm{pda})_{3}(\mathrm{HCOO})_{2}(\text { phen })_{2}\right]$

\section{(d) Mixed-ligand- complexes}

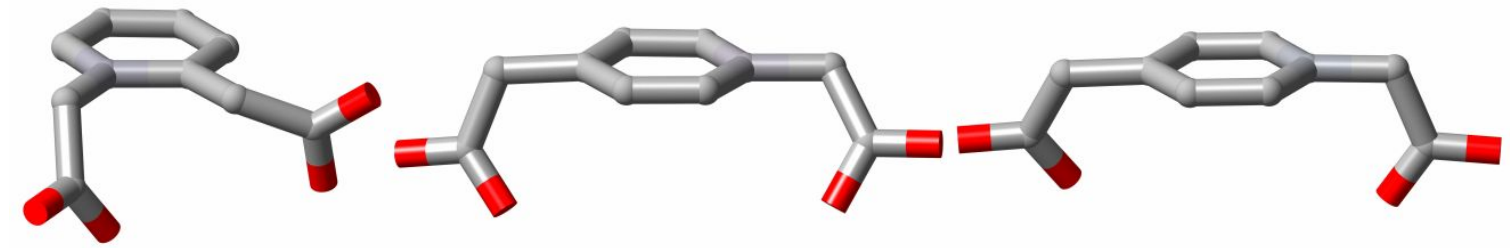

ligand 1

ligand 2

ligand 3

$\left[\mathrm{H}_{2} \mathrm{NMe}_{2}\right]_{2}\left[\left(\mathrm{UO}_{2}\right)_{2}(1,2\right.$-pda $\left.)(1,4 \text {-pda })_{2}\right](\mathbf{1 1})$

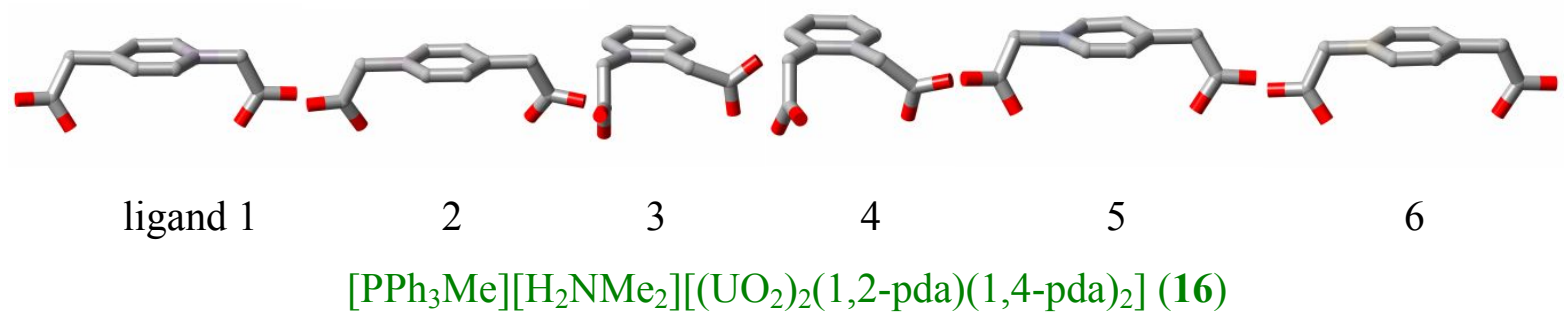




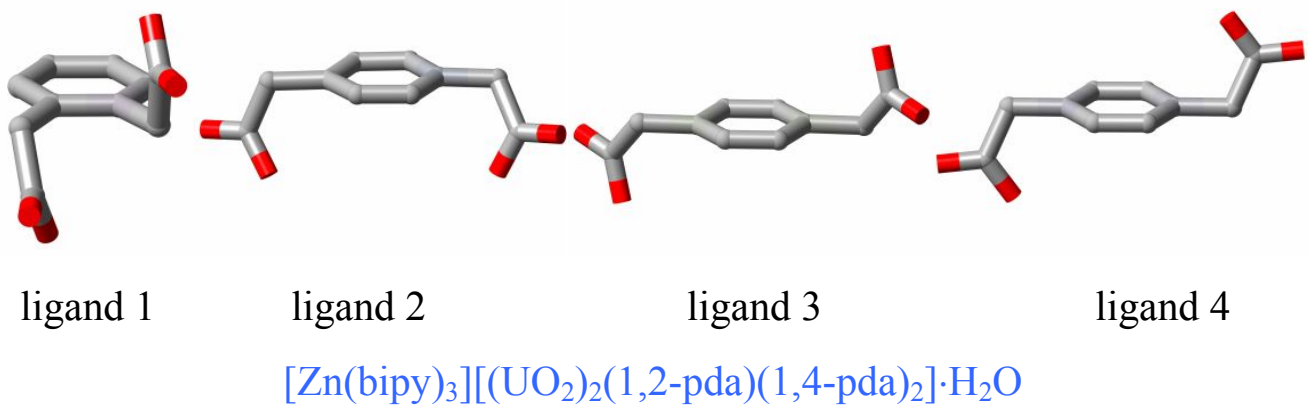

Figure S1 Conformations of inequivalent phenylenediacetate $\left(\mathrm{pda}^{2-}\right)$ units within the structures of uranyl ion complexes. All views with the phenyl ring tilted at $20^{\circ}$ to the line of sight of the viewer and with $\mathrm{CH}_{2} \cdots \mathrm{CH}_{2}$ axes horizontal.

Colour coding for complex names:

Red = P. Thuéry, Y. Atoini, J. Harrowfield, Inorg. Chem., 2019, 58, 6550.

Blue = P. Thuéry, Y. Atoini, J. Harrowfield, Cryst. Growth Des., 2019, 19, 6611.

Green $=$ present work.
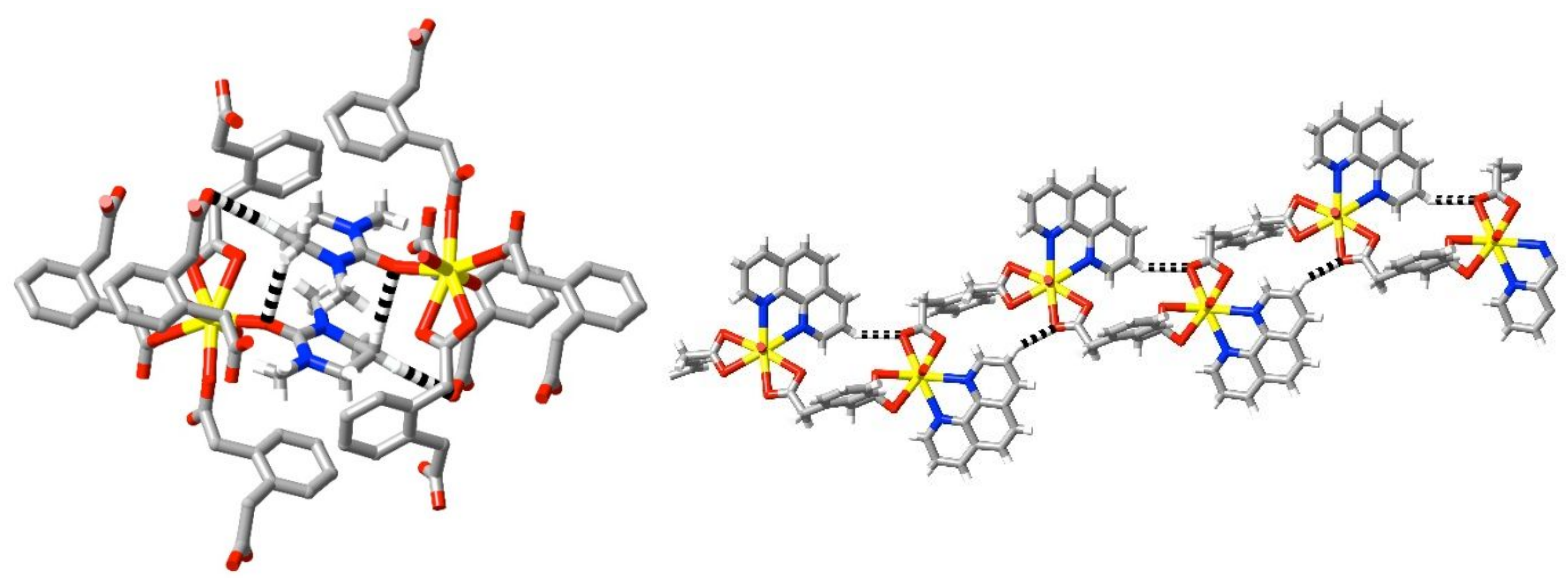

Figure S2 Left: DMPU interactions in $\left[\mathrm{UO}_{2}(1,2-\mathrm{pda})(\mathrm{DMPU})\right]$ (3). Right: phen interactions in $\left[\mathrm{UO}_{2}(1,2-\mathrm{pda})(\mathrm{phen})\right]$. 


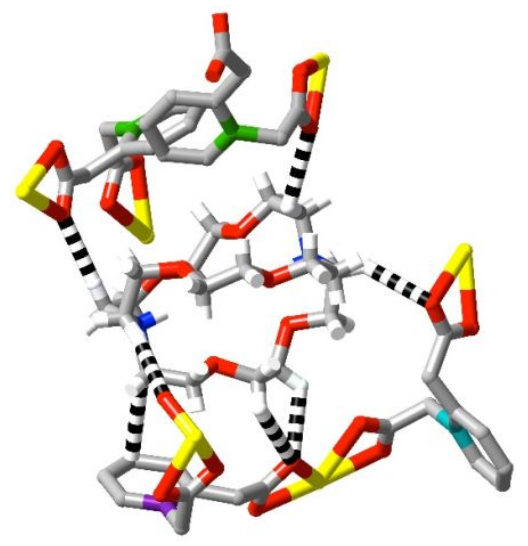

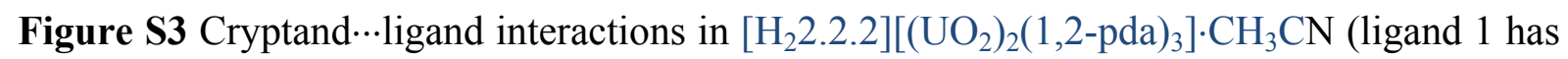
a blue $\mathrm{C}$, ligand 2 a violet and ligand 3 a green).

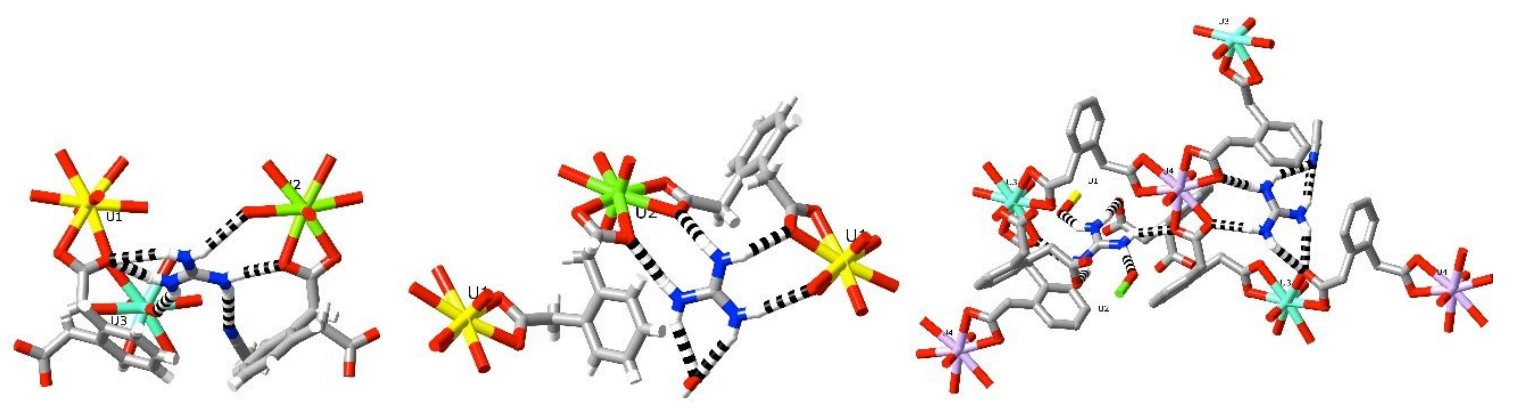

Figure $\mathbf{S 4}$ Interactions of guanidinium cations in $\left[\mathrm{C}\left(\mathrm{NH}_{2}\right)_{3}\right]_{2}\left[\left(\mathrm{UO}_{2}\right)_{2}(1,2-\right.$ $\left.\mathrm{pda})_{3}\right] \cdot 0.5 \mathrm{H}_{2} \mathrm{O} \cdot \mathrm{CH}_{3} \mathrm{CN}(12)$.
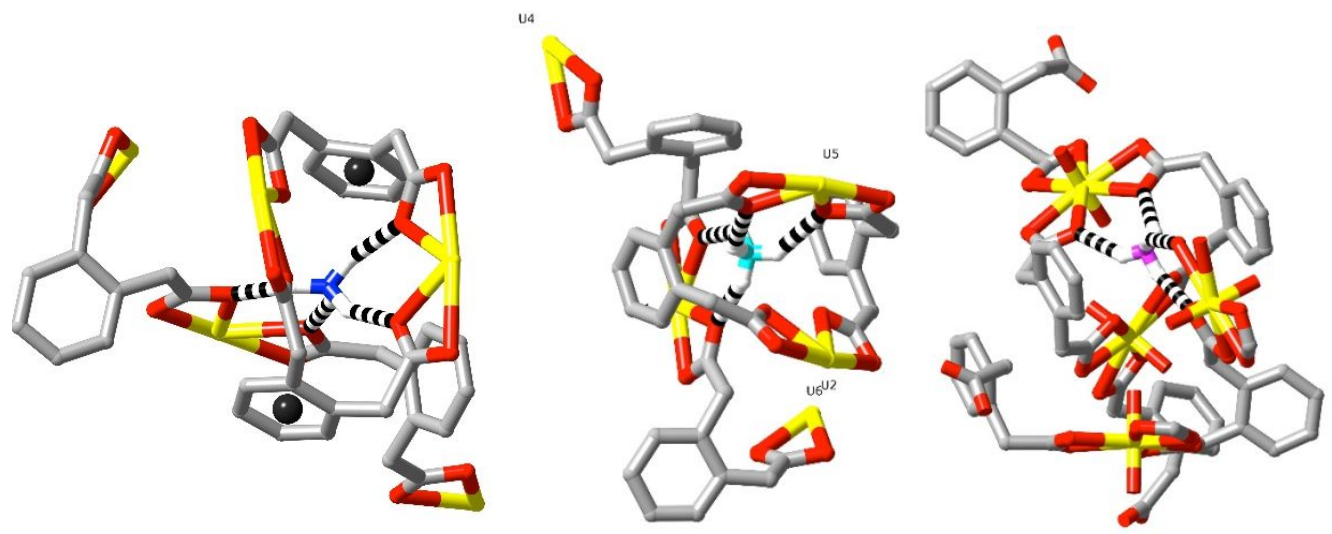

Figure S5 Interactions of ammonium cations in $\left[\mathrm{NH}_{4}\right]_{6}\left[\mathrm{Ni}\left(\mathrm{H}_{2} \mathrm{O}\right)_{6}\right]_{2}\left[\left(\mathrm{UO}_{2}\right)_{4}(1,2-\right.$ pda $\left.)_{6}\right]_{2}\left[\left(\mathrm{UO}_{2}\right)_{4}(1,2-\mathrm{pda})_{5}\left(\mathrm{H}_{2} \mathrm{O}\right)_{4}\right]$. 